\title{
A note on Riemannian connections with skew torsion and the de Rham splitting *
}

\author{
G. Dileo and A. Lotta
}

\begin{abstract}
We prove that a Riemannian manifold admitting a metric connection with totally skew-symmetric torsion and reducible holonomy is locally reducible, provided it has nonpositive sectional curvature.
\end{abstract}

Keywords: connection with skew-symmetric torsion; holonomy representation; de Rham decomposition.

Mathematics Subject Classification (2010): 53C29, 53C05.

\section{Introduction}

The aim of this note is to prove a version of the de Rham splitting theorem for Riemannian manifolds, involving the holonomy of metric connections with totally skew-symmetric torsion. It is well known that such a result cannot hold in full generality; indeed there exist many irreducible Riemannian manifolds admitting a metric connection with totally skew-symmetric torsion and with reducible holonomy. Typically such a connection is adapted to some relevant geometric structure: for instance, every odd dimensional round sphere $S^{2 n+1}$ carries a standard Sasakian structure, which is preserved by exactly one "characteristic connection" $\nabla$ (cf. e.g. [1]). In particular, the contact distribution is $\nabla$-parallel, so that the holonomy representation of $\nabla$ is reducible. One can also mention the Allof-Wallach homogeneous spaces and irreducible 3-Sasakian manifolds, admitting a codimension 3 distribution preserved by a distinguished connection with skew torsion (see $[2,3]$ ). Remarkable examples in even dimension are given by irreducible nearly Kähler manifolds with special algebraic torsion [5], in which case the unique Hermitian connection with skew-symmetric torsion has reducible holonomy.

We shall prove instead that for spaces with nonpositive curvature the situation is completely different, our main result being the following:

\footnotetext{
*Preprint version. Published source: Manuscripta Math. 156, 299-302 (2018). https://doi.org/10.1007/s00229-017-0967-y
} 
Theorem 1.1 Let $(M, g)$ be a connected Riemannian manifold with nonpositive sectional curvature. Let $\nabla$ be a metric connection with totally skewsymmetric torsion. If the holonomy representation of $\nabla$ is reducible, then $M$ is locally a Riemannian product.

The curvature assumption can also be slightly weakened considering manifolds with partially nonpositive curvature, see Theorem 2.2 below.

It is worthwhile to mention some consequences of this result, whose proof is standard. All manifolds are assumed to be connected.

Corollary 1.2 Let $(M, g)$ be an odd dimensional, irreducible Riemannian manifold with nonpositive sectional curvature. Consider a metric connection $\nabla$ with totally skew-symmetric torsion. Then $M$ does not admit any non null $\nabla$-parallel 2-form.

Taking again into account the Sasakian structure of odd dimensional spheres, we see that the curvature assumption in the above corollary is essential.

Corollary 1.3 Let $(M, g)$ be an irreducible Riemannian manifold with nonpositive sectional curvature. If $M$ admits a metric connection $\nabla$ with totally skew-symmetric torsion such that $\nabla \mathrm{Ric}^{g}=0$, then $M$ is Einstein.

We remark that this corollary applies in the special case where $M$ is a locally homogeneous Riemannian manifold admitting an Ambrose-Singer connection $\nabla$ with totally skew-symmetric torsion (cf. $[8,9])$. Actually, in this case more is known, namely $M$ must be locally symmetric. Indeed, under the assumption of nonpositive curvature, a result of Spiro ensures that $M$ is locally isometric to a naturally reductive homogeneous Riemannian manifold, which must be a symmetric space according to paper [4] by Gordon and Ziller.

\section{Proof of the result}

We begin with some basic information concerning metric connections with torsion. For further details, see [1]. Let $(M, g)$ be a Riemannian manifold and let $\nabla$ be a metric connection with torsion $T$. We recall that $\nabla$ is said to have (totally) skew-symmetric torsion if the $(0,3)$-tensor field $T$ defined by

$$
T(X, Y, Z)=g(T(X, Y), Z)
$$

is a 3 -form. In this case the relation between $\nabla$ and the Levi-Civita connection $\nabla^{g}$ is

$$
\nabla_{X} Y=\nabla_{X}^{g} Y+\frac{1}{2} T(X, Y) .
$$


In the following, we denote by $R^{g}$ the Riemannian curvature tensor of $g$ and by $R$ the curvature of $\nabla$. Our proof relies on the following preliminary result, concerning $\nabla$-parallel distributions.

Proposition 2.1 Let $\nabla$ be a metric connection with totally skew-symmetric torsion $T$ on a Riemannian manifold $(M, g)$. If $D$ is a $\nabla$-parallel distribution, then for every $x \in M$ and for every unit tangent vectors $X \in D_{x}$ and $Y \in D_{x}^{\perp}$, the sectional curvature $K^{g}(X, Y)$ of the 2-plane spanned by $X$ and $Y$ is given by:

$$
K^{g}(X, Y)=\frac{1}{4} g(T(X, Y), T(X, Y)) \geq 0 .
$$

Proof. We recall that the curvature tensor of $\nabla$ is given by

$$
R(X, Y) Z=R^{g}(X, Y) Z+B(X, Y) Z,
$$

where

$$
\begin{aligned}
B(X, Y) Z= & -\frac{1}{4} T(X, T(Y, Z))+\frac{1}{4} T(Y, T(X, Z))+\frac{1}{2} T(T(X, Y), Z) \\
& +\frac{1}{2}\left(\nabla_{X} T\right)(Y, Z)-\frac{1}{2}\left(\nabla_{Y} T\right)(X, Z) .
\end{aligned}
$$

Now, since $T$ is totally skew-symmetric, we have

$$
\begin{aligned}
g(B(X, Y) Y, X) & =\frac{1}{4} g(T(T(X, Y), Y), X)-\frac{1}{2} g\left(\left(\nabla_{Y} T\right)(X, Y), X\right) \\
& =-\frac{1}{4} T(X, Y, T(X, Y)) \\
& =-\frac{1}{4} g(T(X, Y), T(X, Y)) .
\end{aligned}
$$

Being $D$ and $D^{\perp}$ both parallel with respect to $\nabla$, we also have $g(R(X, Y) Y, X)=$ 0 and (1) follows.

We are now ready to prove Theorem 1.1. We shall actually provide a proof of a more general statement. Recall that, given a Riemannian manifold $(M, g)$ of dimension $n \geq 2$, fixing an integer $k, 1 \leq k \leq n-1$, then $M$ is said to have nonpositive $(n-k)$-Ricci curvature provided for every $x \in M$ and for every mutually orthogonal unit tangent vectors $\left\{E_{0}, E_{1}, \ldots, E_{k}\right\}$ in $T_{x} M$ we have

$$
\sum_{i=1}^{k} K^{g}\left(E_{0}, E_{i}\right) \leq 0
$$

(cf. e.g. [6]). Observe that nonpositive 1-Ricci curvature means nonpositive sectional curvature, while nonpositive $(n-1)$-Ricci curvature means nonpositive Ricci curvature. 
Theorem 2.2 Let $(M, g)$ be a connected Riemannian manifold of dimension $n$ and let $\nabla$ be a metric connection with totally skew-symmetric torsion and reducible holonomy. Let $k$ be the minimum dimension of a non null $\mathrm{Hol}^{\nabla}\left(x_{o}\right)-$ invariant subspace of the tangent space at some point $x_{o} \in M$. If $M$ has nonpositive $(n-k)$-Ricci curvature, then $M$ is locally a Riemannian product.

Proof. Let $D$ be the rank $k$ distribution on $M$ obtained from a $k$-dimensional $\mathrm{Hol}^{\nabla}\left(x_{o}\right)$-invariant subspace of $T_{x_{o}} M$ by parallel transport with respect to $\nabla$. We claim that $K^{g}(X, Y)=0$ for every $x \in M$ and for every unit vectors $X \in D_{x}$ and $Y \in D_{x}^{\perp}$. Indeed, choose an orthonormal basis $\left\{Y_{1}, \ldots, Y_{n-k}\right\}$ of $D_{x}^{\perp}$ with $Y_{1}=Y$; then the curvature assumption implies that

$$
\sum_{i=1}^{n-k} K^{g}\left(X, Y_{i}\right) \leq 0 .
$$

On the other hand, since $D$ is $\nabla$-parallel, Proposition 2.1 forces

$$
K^{g}\left(X, Y_{i}\right)=0, \quad i=1, \ldots, n-k,
$$

and the claim follows. Using again (1) we obtain that $T(X, Y)=0$ for every $X \in \Gamma D$ and $Y \in \Gamma D^{\perp}$, and thus

$$
\nabla_{X}^{g} Y=\nabla_{X} Y \in \Gamma D^{\perp}, \quad \nabla_{Y}^{g} X=\nabla_{Y} X \in \Gamma D .
$$

Consequently we get

$$
g\left(\nabla_{X^{\prime}}^{g} X, Y\right)=-g\left(\nabla_{X^{\prime}}^{g} Y, X\right)=0
$$

for every $X, X^{\prime} \in \Gamma D$ and $Y \in \Gamma D^{\perp}$. We conclude that $D$ is $\nabla^{g}$-parallel so that the classical de Rham theorem applies.

We conclude with the following remark concerning the four dimensional case.

Corollary 2.3 Let $(M, g)$ be a connected Riemannian manifold of dimension 4 with nonpositive sectional curvature and negative definite Ricci tensor. If $\nabla$ is a metric connection with totally skew-symmetric torsion and reducible holonomy, then $\nabla$ is the Levi-Civita connection.

Proof. Since $K^{g} \leq 0$, by the above result, $M$ is locally a Riemannian product $M_{1} \times M_{2}$. Letting $T$ be the torsion of $\nabla$, we obtain that $T(X, Y)=0$ for every $X$ tangent to $M_{1}$ and $Y$ tangent to $M_{2}$. Now, Ric $^{g}<0$ implies $\operatorname{dim}\left(M_{1}\right)=\operatorname{dim}\left(M_{2}\right)=2$ and this suffices to conclude that $T=0$. 


\section{References}

[1] I. Agricola: The Srní lectures on non-integrable geometries with torsion, Arch. Math. (Brno) 42 (2006), suppl., 5-84.

[2] I. Agricola, T. Friedrich: On the holonomy of connections with skewsymmetric torsion, Math. Ann. 328 (2004), no. 4, 711-748.

[3] I. Agricola, G. Dileo: New classes of almost 3-contact metric manifolds and their remarkable connections, preprint (2017).

[4] C. Gordon, W. Ziller: Naturally reductive metrics of nonpositive Ricci curvature, Proc. Amer. Math. Soc. 91 (1984), no. 2, 287-290.

[5] P.A. Nagy: Nearly Kähler geometry and Riemannian foliations, Asian J. Math. 6 (2002), no. 3, 481-504.

[6] Y. V. Rovenskii: Foliations on Riemannian manifolds and submanifolds, Birkhäuser Boston, Inc., Boston, MA, 1998.

[7] A. Spiro: A remark on locally homogeneous Riemannian spaces, Results Math. 24 (1993), no. 3-4, 318-325.

[8] F. Tricerri: Locally homogeneous Riemannian manifolds, Differential geometry (Turin, 1992). Rend. Sem. Mat. Univ. Politec. Torino 50 (1992), no. 4, 411-426 (1993).

[9] F. Tricerri, L. Vanhecke: Homogeneous structures on Riemannian manifolds, London Mathematical Society Lecture Note Series 83, Cambridge University Press, Cambridge, 1983.

Giulia Dileo

Dipartimento di Matematica, Università degli Studi di Bari Aldo Moro

Via E. Orabona 4, 70125 Bari, ITALY

E-mail address: giulia.dileo@uniba.it

Antonio Lotta

Dipartimento di Matematica, Università degli Studi di Bari Aldo Moro

Via E. Orabona 4, 70125 Bari, ITALY

E-mail address: antonio.lotta@uniba.it 